Introduction

\title{
La traite saisie par les institutions : entre contrôle des frontières et gouvernement des sexualités
}

\section{Mathilde Darley}

\section{(2) OpenEdition \\ Journals}

\section{Édition électronique}

URL : https://journals.openedition.org/conflits/22819

DOI : $10.4000 /$ conflits.22819

ISSN : $1777-5345$

Éditeur :

CECLS - Centre d'études sur les conflits - Liberté et sécurité, L'Harmattan

Édition imprimée

Date de publication : 25 octobre 2021

Pagination : 7-17

ISBN : 978-2-343-24627-7

ISSN : $1157-996 \mathrm{X}$

\section{Référence électronique}

Mathilde Darley, «La traite saisie par les institutions : entre contrôle des frontières et gouvernement des sexualités ", Cultures \& Conflits [En ligne], 122 | été 2021, mis en ligne le 25 octobre 2021, consulté le 02 novembre 2021. URL : http://journals.openedition.org/conflits/22819 ; DOI : https://doi.org/ $10.4000 /$ conflits.22819 


\title{
La traite saisie par les institutions : entre contrôle des frontières et gouvernement des sexualités
}

\author{
Introduction
}

\section{Mathilde DARLEY}

Mathilde Darley est chargée de recherche CNRS au Cesdip (Centre de recherches sociologiques sur le droit et les institutions pénales), directrice adjointe $d u$ Cesdip et chercheuse associée an Centre Marc Bloch (Berlin). Ses thèmes de recherche incluent le contrôle de l'immigration, l'enfermement des étrangers, la prostitution et la traite des êtres humains, la sociologie de la police et de la justice pénale. Elle a coordonné entre 2014 et 2018 le projet franco-allemand ProsCrim (ANR-DFG) portant sur la traite des êtres humains et le proxénétisme.

$\mathrm{E}$ ntre le début des années 1990 et le tournant des années 2000, la traite des êtres humains, entendue comme « le recrutement, le transport, le transfert, l'hébergement ou l'accueil de personnes, par la menace de recours ou le recours à la force ${ }^{1}$ ", et en particulier la traite à des fins d'exploitation sexuelle, est (re)devenue 2 un enjeu international majeur. Si les chiffres censés attester de l'ampleur du phénomène présentent des variations importantes (les estimations des organisations internationales varient de 700000 à 4 millions de victimes par an ${ }^{3}$ ), ils n'ont toutefois pas entravé la constitution d'un consensus

1. Voir la définition du Protocole de Palerme, additionnel à la Convention des Nations Unies contre la criminalité transnationale organisée visant à prévenir, réprimer et punir la traite des personnes, en particulier des femmes et des enfants (adopté le 15 novembre 2000 par l'Assemblée Générale des Nations Unies), à savoir : « le recrutement, le transport, le transfert, l'hébergement ou l'accueil de personnes, par la menace de recours ou le recours à la force ou à d'autres formes de contrainte, par enlèvement, fraude, tromperie, abus d'autorité ou d'une situation de vulnérabilité, ou par l'offre ou l'acceptation de paiements ou d'avantages pour obtenir le consentement d'une personne ayant autorité sur une autre aux fins d'exploitation ».

2. Sur la construction de la traite des femmes en problème public international au début du XXe siècle, voir Chaumont J.-M., Le mythe de la traite des blanches. Enquête sur la fabrication d'un fléau, Paris, La Découverte, 2009.

3. À propos du caractère problématique de la production de ces données chiffrées, voir Kelly L., "'You can find anything you want': A Critical Reflection on Research on Trafficking in Persons within and into Europe”, International Migration, vol. 43, n¹/2, 2005, pp. 235-265; 
politique, juridique et médiatique autour de la nécessité de déployer de nouveaux instruments juridiques et politiques pour lutter contre la traite. Ce consensus quant à la nécessité d'agir, porté par des acteurs étatiques et non étatiques, nationaux et internationaux, pénaux et sociaux, s'est traduit par une inflation normative et juridique que reflètent notamment, depuis le début des années 1990, la multiplication des textes internationaux et leur traduction en droit national ${ }^{4}$. Ainsi, après le Protocole sur la traite, dit "Protocole de Palerme ", adopté par les Nations Unies en novembre 2000 dans le cadre de la Convention contre la criminalité organisée, puis la décision-cadre sur la lutte contre la traite du Conseil de l'Europe en 2005, et enfin la directive du Parlement et du Conseil européens en $2011^{5}$, la qualification juridique de traite des êtres humains est finalement introduite dans le Code pénal français en $2013{ }^{6}$.

Pourtant, le consensus apparent quant à la nécessité de lutter contre la traite, présentée comme le nouveau visage de «l'esclavage moderne », ne saurait masquer les querelles interprétatives entourant la définition $\mathrm{du}$ " problème » et ses origines supposées. La traite des êtres humains apparaît en effet comme un espace où s'enkystent et se prolongent d'autres controverses contemporaines, relatives notamment à la sexualité et à sa tarification, aux migrations internationales ou à la criminalité organisée 7 .

La traite, et en particulier la traite à des fins d'exploitation sexuelle, donne ainsi d'abord et avant tout prise aux sex wars ${ }^{8}$ qui, depuis les débuts de la régulation de la prostitution à la fin du XIXe siècle 9 , traversent la question prostitutionnelle. À partir des années 1970, la question prostitutionnelle clive en effet jusqu'au mouvement féministe lui-même ${ }^{10}$, selon une ligne de fracture autour de laquelle la plupart des analyses continuent aujourd'hui encore de se positionner : aux féministes radicales, ou abolitionnistes, pour lesquelles

Tyldum G., Brunovskis A., "Describing the Unobserved: Methodological Challenges in Empirical Studies on Human Trafficking”, International Migration, vol. 43, n¹/2, 2005, pp. 17-34; Mathieu L., « De l'objectivation à l'émotion. La mobilisation des chiffres dans le mouvement abolitionniste contemporain ", Mots. Les langages du politique, $\mathrm{n}^{\circ} 100,2012$, pp. 173-185.

4. Halley J., Kotiswaran P., Shamir H., Thomas C., "From the International to the Local in Feminist Legal Responses to Rape, Prostitution/Sex Work and Sex Trafficking”, Harvard Journal of Law E Gender, vol. 29, n², 2006, pp. 336-423; Ragaru N., «Du bon usage de la traite des êtres humains. Controverses autour d'un problème social et d'une qualification juridique ", Genèses, n 66,2007, pp. 69-89.

5. Directive 2011/36/U6.

6. Article 225-4-1. - I du Code pénal.

7. Ragaru N., op. cit.

8. Ferguson A., "Sex war: The Debate between Radical and Libertarian Feminists", Signs, n¹0, 1989, pp. 106-112.

9. Corbin A., Les filles de noce : misère sexuelle et prostitution, XIXe et XXe siècles, Paris, Flammarion, 1982.

10. Vance C. S. (ed.), Pleasure and Danger: Exploring Female Sexuality, Boston/London, Routledge \& Kegan Paul, 1984 ; Weitzer R., "The Growing Moral Panic over Prostitution and Sex Trafficking”, The Criminologist, vol. 30, n5, 2005, pp. 2-5. 
la prostitution constituerait une expression exacerbée de la domination masculine, s'opposent les féministes libertaires, aux yeux desquelles la prostitution (comme la pornographie ou d'autres pratiques socialement construites comme "déviantes ") pourrait constituer un moyen de reconquête, par les femmes, de leur sexualité, à travers la levée des censures imposées par une société patriarcale sexuellement répressive ${ }^{11}$. La virulence des débats et le " pouvoir émotionnel 12 » de l'exploitation sexuelle, expliquent ainsi, pour partie au moins, que l'exploitation de la prostitution ait bien davantage retenu l'attention que d'autres formes d'exploitation (travail forcé, mendicité, extraction d'organes, etc.) pourtant incluses dans la définition de la traite. Sans surprise, dans un domaine où monde académique et cercles militants se recoupent pour partie, ces antagonismes autour de la question du sexe tarifé se rejouent dans des débats de sciences sociales, longtemps marqués par la sur-représentation de deux corpus d'analyses 13 : tandis que les écrits s'inspirant de l'approche abolitionniste mettaient l'accent sur la vulnérabilité de la victime, jeune femme étrangère qu'il conviendrait de "sauver ${ }^{14}$ », d'autres travaux revendiquant une approche " pro-droits » soulignaient l'agency ${ }^{15}$, ou « puissance d'agir 16 », dont sont dotées les prostituées migrantes, pour lesquelles le travail du sexe constituerait une « ressource migratoire 17 » dans un contexte politique entravant leur liberté de circulation.

Car si la traite à des fins prostitutionnelles est au cour de luttes idéologiques ayant trait aux droits des femmes et à la sexualité tarifée, elle croise également les débats relatifs aux migrations internationales ${ }^{18}$ et à leur criminalisation: ainsi, la plupart des instruments internationaux adoptés au cours des dernières décennies pour lutter contre la traite sont de facto ancrés dans la lutte contre la criminalité organisée (à commencer par le Protocole de Palerme annexé à la Convention des Nations Unies sur la criminalité organisée) et la protection de la souveraineté des États, davantage que dans un référentiel normatif de défense des droits humains ${ }^{19}$. De même, dans les débats parlemen-

11. Mathieu L., La fin du tapin. Sociologie de la croisade pour l'abolition de la prostitution, Lormond, Éd. François Bourin, 2014, p. 63.

12. Corbin A., op. cit., p. 405.

13. Jakšić M., «État de littérature. Déconstruire pour dénoncer : la traite des êtres humains en débat », Critique internationale, vol. 4, n53, 2011, pp. 169-182.

14. Sur cette « industrie du sauvetage » à destination des travailleuses du sexe migrantes, voir notamment Agustín L., Sex at the Margins. Migration, Labour, Markets and the Rescue Industry, Londres, Zed Books, 2007.

15. Andrijaševic R., Migration, Agency and Citizenship in Sex Trafficking, New York, Palgrave Macmillan, 2010.

16. Guillemaut F., «Victimes de trafic ou actrices d'un processus migratoire ? Saisir la voix des femmes migrantes prostituées par la recherche-action ", Terrains $E$ Travaux, vol. 1, $\mathrm{n}^{\circ} 10$, 2006, pp. 157-176.

17. Lévy F., Lieber M., "La sexualité comme ressource migratoire. Les Chinoises du Nord de Paris », Revue française de sociologie, vol. 50, n4, 2009, pp. 719-746.

18. Bien que la définition donnée par le Protocole de Palerme adopté par l'ONU en 2000 ne pose pas le franchissement des frontières comme un élément de définition de la traite.

19. Aradau C., Rethinking Trafficking in Women. Politics out of Security, New York, Palgrave 
taires nationaux ${ }^{20}$, les arguments anti-traite ont généralement été mobilisés en vue de durcir la lutte contre l'immigration clandestine en distinguant les travailleuses du sexe «nationales » des étrangères (sans-papiers) et ce, au nom d'une figure idéalisée de victime en souffrance et à sauver. Cette rhétorique humanitaire semble en effet trouver un point d'ancrage particulier, en matière de certification de la souffrance, dans le déploiement de la figure de victime de violences sexuelles en général, et de victime de traite en particulier, que l'atteinte à son intégrité sexuelle et la convocation d'un imaginaire de l'esclavage constituent en "victime absolue 21 ». Le cas des prostituées étrangères offre toutefois à cet égard un intérêt particulier : si elles constituent une surface de projection privilégiée, tant dans la sphère médiatique et politique que scientifique, aux représentations stéréotypiques d'une «victime » et à la politique compassionnelle censée les accompagner ${ }^{22}$, leur appréhension ne parvient toutefois pas à s'émanciper des termes sécuritaires du débat public autour des questions d'immigration et de prostitution ${ }^{23}$.

La pathologisation et la criminalisation, en termes de traite et d'exploitation, de certaines formes de migrations liées au genre et/ou à la sexualité débouchent ainsi sur un double processus d'humanitarisation des discours ${ }^{24}$, adossé à une altérisation et une essentialisation ethnoraciales et sexuelles des

Macmillan, 2008 ; Darley M., «Le statut de la victime dans la lutte contre la traite des femmes », Critique internationale, n³0, 2006, pp. 103-122.

20. Pareilles analyses ont ainsi été déployées en Grande-Bretagne, aux Pays-Bas, aux États-Unis ou en France. Voir Kantola J., Squires J., "Discourses Surrounding Prostitution Policies in the UK", European Journal of Women's Studies, vol. 11, n¹, 2004, pp. 77-101 ; Outshoorn J., "Debating Prostitution in Parliament: A Feminist Analysis", European Journal of Women's Studies, vol. 8, n4, 2001, pp. 472-490 ; Outshoorn Joyce, "The Political Debates on Prostitution and Trafficking in Women", Social Politics: International Studies in Gender, State and Society, vol. 12, n¹, 2005, pp. 141-155; Chapkis W., "Trafficking, Migration and the Law: Protecting Innocents, Punishing Immigrants", Gender and Society, vol. 17, n6, 2003, pp. 923-937 ; Soderlund G., "Running from the Rescuers: New U.S. Crusades against Sex Trafficking and the Rhetoric of Abolition", NWSA Journal, vol. 17, n³, 2005, pp. 64-87; Zimmermann Y. C., "From Bush to Obama. Rethinking Sex and Religion in the United States' Initiative to Combat Human Trafficking”, Journal of Feminist Studies in Religion, vol. 26, n`1, 2010, pp. 79-99; Jakšić M., La traite des êtres humains en France. De la victime idéale à la victime coupable, Paris, CNRS Éditions, 2016 ; Jacquemart A., Jakšić M., « Droits des femmes ou femmes sans droits ? Le féminisme d'État face à la prostitution ", Genre, sexualité E société, n²0, 2018 [http://journals.openedition.org/gss/5006], page consultée le 28 septembre 2021.

21. Mai N., op. cit.

22. Fassin D., «La souffrance du monde. Considérations anthropologiques sur les politiques contemporaines de la compassion ", Évolution psychiatrique, vol. 67, n 4, 2002, pp. 676-689; Barbot J., Dodier N., « De la douleur au droit. Ethnographie des plaidoiries lors de l'audience pénale du procès de l'hormone de croissance contaminée ", in Cefaï D., Berger M., GayetViaud C. (dir.), Du civil au politique. Ethnographies du vivre ensemble, Bruxelles, Peter Lang, 2011, pp. 289-322.

23. Jakšsic M., La traite des êtres humains..., op. cit.

24. Nick Mai parle à ce sujet d'« humanitarisme sexuel ». Voir Mai N., "Between Embodied Cosmopolitism and Sexual Humanitarianism. The Fractal Mobilities and Subjectivities of Migrants Working in the Sex Industry", in Anteby-Yemini L., Baby-Collins V., Mazzella S. (eds.), Borders, Mobilities and Migrations, Perspectives from the Mediterranean in the 21st Century, Bruxelles, Peter Lang, 2014. 
victimes ${ }^{25}$, et d'hostilité déclarée à l'encontre des « réseaux criminels » censés organiser leur exploitation. C’est ce « grand récit 26 » qui guide la politisation contemporaine de la traite dans les sociétés ouest-européennes, justifiant par la protection des victimes le déploiement d'une politique répressive de renforcement du contrôle aux frontières et de lutte contre l'immigration clandestine ${ }^{27}$.

Toutefois, si les grandes lignes du régime d'énonciation de la traite en enjeu de politique publique sont désormais bien connues, leurs déclinaisons pratiques, et notamment leurs effets sur la production, en actes, des figures tutélaires de « victime » et « trafiquant », « proxénète » ou « exploiteur », restent moins appréhendés ${ }^{28}$. C'était donc l'objet du projet ProsCrim ${ }^{29}$ que de proposer une analyse empiriquement informée des registres d'interaction entre, d'une part, étrangères et étrangers impliqués dans des formes de sexualité prostitutionnelle et, d'autre part, institutions chargées de leur contrôle et/ou de leur prise en charge, et donc de leur « labellisation ». Autrement dit, plutôt que de s'intéresser aux «victimes » et aux « exploiteurs » en tant que groupes sociaux identifiés, le programme ProsCrim visait bien plutôt à mettre en lumière les processus de catégorisation au terme desquels ces figures se trouvent institutionnellement produites et consolidées.

Pour ce faire, il s'agissait d'abord d'élargir la focale, jusqu'alors majoritairement concentrée soit sur l'énonciation des politiques de lutte contre la traite, soit sur les discours et les parcours des femmes prostituées, souvent dépeints en termes « misérabilistes » ou " populistes 30 ». La première perspective, relevant d'une démarche de recherche dite macrosociale, présentait le risque de centrer l'analyse sur l'État comme lieu privilégié du pouvoir ; la seconde au contraire, dite microsociale, risquait d'occulter, pour partie au moins, la question des rapports de pouvoir ${ }^{31}$ et mécanismes d'assignation, à travers les interactions dans lesquelles ils s'incarnent et se réalisent. Tout en prenant acte des apports des travaux existants, l'approche revendiquée ici vise donc à com-

25. Tiktin M., "Sexual Violence as the language of Border Control: Where French Feminist and Anti-Immigrant Rhetoric Meet”, Signs: Journal of Women and Culture in Society, vol. 33, $\mathrm{n}^{\circ} 4$, pp. 863-889.

26. Snajdr E., "Beneath the master narrative: Human trafficking, myths of sexual slavery and ethnographic realities”, Dialectical Anthropology, vol. 37, n², 2013, pp. 229-256.

27. Mathieu L., "An ambiguous compassion: Policing and debating prostitution in contemporary France”, Sexuality Research and Social Policy, vol. 9, n³, 2012, pp. 203-211 ; Jakšić M., La traite des êtres humains..., op. cit.

28. Pour une analyse de la manière dont les catégories juridiques relatives à la prostitution et son exploitation se trouvent opérationnalisées dans les interactions quotidiennes entre la police et ses publics-cibles, voir Mainsant G., Sur le trottoir, l'État, Paris, Seuil, 2021.

29. Projet franco-allemand ProsCrim - La traite des êtres humains saisie par les institutions, financé par l'ANR (ANR-13-FRAL-0014-01) de 2014 à 2018 (coord. Mathilde Darley) et par la DFG de 2014 à 2017 (coord. Rebecca Pates).

30. Mathieu L., La condition prostituée, Paris, Textuel, 2007.

31. Bayart J.-F., Warnier J.-P. (dir.), Matière à politique. Le pouvoir, les corps et les choses, Paris, Karthala, 2004, p. 28-29. 
plexifier l'analyse des politiques de lutte contre l'exploitation sexuelle découlant soit de l'étude du « grand récit » de la traite, soit de celle des trajectoires migratoires ou prostitutionnelles. En portant une attention particulière à une sociologie des acteurs et des institutions en interaction, il s'agit en outre de dépasser la classique dichotomie entre "approche par le haut » et « approche par le bas » de l'action publique pour mettre en évidence la « construction d'espaces d'action publique mêlant des acteurs aux logiques, aux intérêts, aux pratiques et aux représentations différentes $32 »$. Nous proposons ainsi, dans ce numéro, une analyse croisée des différents « intermédiaires » de l'action publique que sont tout à la fois les professionnels du régalien (policiers et magistrats en particulier), les acteurs sociaux (et notamment associatifs), mais aussi plus largement les passeurs de frontières (physique ou administrative), porteurs de discours, de représentations, de savoirs et de savoir-faire contribuant à «faire exister » la traite (ses auteurs et ses victimes) et à façonner les formes de régulation mises en œuvre pour y répondre. L'attention accordée aux valeurs, affects et croyances normatives de ces acteurs en interaction, et aux circulations de savoirs et de pratiques entre les différents maillages de l'action publique qu'ils incarnent (répressifs ou assistanciels, publics ou privés, etc.), esquisse ici les contours d'une construction collective de la traite comme problème social, présidant à la mise en récit de l'action publique ${ }^{33}$.

À partir de données empiriques (observations, entretiens, consultation de dossiers) collectées dans l'arène judiciaire, policière et associative, les textes réunis ici entendent donc poursuivre une réflexion commune visant à questionner la traduction, dans les pratiques institutionnelles et les expériences individuelles, des catégories d'action énoncées par les dispositifs locaux, nationaux et internationaux de lutte contre la traite. Cherchant notamment à interroger et complexifier la dichotomie «victimes » / «trafiquants» traversant le débat public sur l'exploitation sexuelle, la série d'articles proposés dans ce dossier s'ouvre sur l'expérience des étrangères en situation de prostitution. À partir d'une longue enquête conduite auprès de travailleuses du sexe au sein d'une association venant en aide aux victimes de traite, Prune de Montvalon propose ainsi une analyse des trajectoires de prostituées migrantes sortant de la dichotomie évoquée plus haut, et qui revient généralement à aplatir leurs parcours sous un prisme soit misérabiliste (faisant d'elles des femmes agies plus qu'agissantes, pensées plus que pensantes) soit résolument héroïsant, soulignant leur autonomie (empowerment) et conduisant souvent à minimiser les contraintes et entraves qui jalonnent leur parcours. Pour ce faire, Prune de Montvalon se concentre sur le "passage » des frontières, les acteurs qu'il engage et les savoirs et savoir-faire qu'il requiert. Loin de cantonner la figure

32. Hassenteufel P., Sociologie politique de l'action publique, Paris, Armand Colin, 2011 (2e édition), p. 112.

33. De Maillard J., Kübler D., Analyser les politiques publiques, Grenoble, Presses Universitaires de Grenoble, 2015 (2e édition), p. 213. 
du « passeur » à la représentation criminalisée qui prévaut, et qui revient à désigner ainsi les acteurs impliqués dans l'aide au franchissement illégal des frontières nationales, Prune de Montvalon propose au contraire un exercice d'abstraction visant à penser ensemble le rôle, dans les trajectoires migratoires et prostitutionnelles, des différentes figures de "passeurs ", officielles ou non, entendues comme l'ensemble des agents permettant aux prostituées migrantes de franchir des frontières. Ces dernières sont envisagées non pas comme les seules limites «physiques » du territoire national, mais comme un entrelacs de mises à l'écart administratives, morales, économiques ou sociales invitant à complexifier la traditionnelle partition entre figures « légales » et «illégales ». Conceptualiser la notion de « passeurs » de frontière au-delà de cette traditionnelle dichotomie permet alors de penser ensemble les offres de protection concurrentes émanant, pour les victimes de traite, à la fois de l'État (sous la forme d'un droit au séjour en cas de reconnaissance du statut de victime, notamment), des acteurs criminalisés du passage clandestin, et des acteurs associatifs engagés dans l'accompagnement des victimes et la défense de leurs droits au séjour. Outre qu'il place au cœur de l'analyse l'épaisseur et la complexité des expériences de la frontière par les prostituées migrantes, ce texte contribue ainsi à dessiner une sociologie compréhensive du continuum d'acteurs impliqués dans les politiques de lutte contre la traite. Par ailleurs, en envisageant les acteurs associatifs comme des "passeurs de frontières », Prune de Montvalon s'autorise à lever un tabou que les liens étroits entre recherche et militantisme, dans le domaine de la traite comme dans d'autres, ont contribué à forger : à rebours d'une sacralisation des pratiques associatives d'assistance aux victimes, le texte de Prune de Montvalon, en recourant à la notion de « passeurs », met au jour les stratégies déployées par les associations pour « conformer» les victimes aux attentes institutionnelles et souligne ainsi, également, leur rôle de «passeurs de normes » (y compris comportementales ou émotionnelles).

C'est précisément aux adaptations professionnelles, discursives et pratiques, qu'impose aux acteurs associatifs la coopération avec les acteurs policiers (et inversement), que s'intéresse le texte d'Anne Dölemeyer et Julia Leser. Les enquêtes sur lesquelles il s'appuie ont été réalisées dans le cadre du projet ProsCrim en Allemagne, où la coopération entre associations et police dans le domaine de la lutte contre la traite et de l'assistance aux victimes s'est trouvée à la fois institutionnalisée et contractualisée. Dans un cadre législatif légalisant le travail du sexe mais interdisant son exploitation en Allemagne, les associations et les pouvoirs policiers sont pourtant mus par des objectifs contrastés : tandis que les premières s'attachent à la défense des droits humains des travailleurs et travailleuses du sexe, les seconds se concentrent sur la poursuite pénale des auteurs d'exploitation. À partir d'observations et d'entretiens auprès des acteurs policiers et des associations liés par un accord de coopération, Anne Dölemeyer et Julia Leser interrogent les conditions et les formes 
d'un rapprochement entre ces deux univers professionnels a priori antagonistes et mettent ainsi à jour des dynamiques partenariales, entre acteurs du social et acteurs du pénal, que leur non-formalisation rend plus souterraines (et donc moins directement saisissables) en France. Car si l'objectif de sanction pénale apparaît central dans les accords de coopération conclus entre associations et pouvoirs policiers, celui-ci ne saurait, dans les formes d'administration de la preuve pénale en Allemagne, être atteint sans le concours de la victime, dont le témoignage constitue l'élément de preuve essentiel ${ }^{34}$. Autrement dit, les accords de coopération sont certes présentés comme au service des poursuites pénales, mais la protection de la victime-témoin en est indissociable. Il est dès lors intéressant de comprendre comment le rapprochement entre acteurs policiers et associatifs initiés via les accords de coopération fait bouger les lignes des cultures professionnelles des deux groupes, et comment se met ainsi en place une action publique de lutte contre la traite co-produite par des acteurs pro-droits et des acteurs répressifs. La conception même des accords est cependant sans ambiguïté quant aux objectifs de l'action publique : s'il s'agit de protéger la victime et ses droits, c'est avant tout pour permettre une procédure pénale permettant de condamner le proxénète / trafiquant. Celui-ci reste ainsi la figure centrale des politiques anti-traite, traversant l'ensemble des univers professionnels (étatiques ou non étatiques) par lesquels elles s'incarnent.

Figure centrale des politiques de lutte contre l'exploitation sexuelle, l'exploiteur, le proxénète ou le trafiquant reste pourtant notoirement sousexploré dans la littérature consacrée aux questions de traite et de proxénétisme. Prenant acte de ce relatif silence académique, le texte de Gilles FavarelGarrigues et Lilian Mathieu entend donner corps à la figure du proxénète en étudiant la manière dont l'audience pénale en dessine les contours. À partir d'observations réalisées au tribunal dans le cadre du projet ProsCrim, les auteurs cherchent à montrer comment les qualifications juridiques s'adossent à d'autres formes de jugements, notamment moraux, sur ce qui fait le «bon proxénète ». Pour ce faire, ils étudient les attentes exprimées par les magistrats, dans leur tentative de cerner la figure du proxénète, et qui portent à la fois sur les conduites individuelles et la personnalité des mis en cause et sur leur inscription plus globale dans une configuration criminelle en " réseau ». L'audience pénale, en tant que lieu où se donnent à voir les frictions et troubles qui accompagnent, chez les professionnels du droit, la définition de l'infraction, donne ainsi à voir comment s'opère le cadrage de la figure du proxénète permettant de subsumer l'hétérogénéité des situations sous une catégorie juridique unique et apparemment stabilisée dans l'arène juridique. L'ethnographie des audiences invite alors notamment à souligner le rôle particulier des représentations de genre dans la consolidation de la figure du proxénète par les professionnels du droit. 
Le texte que je signe, résultant lui aussi des observations d'audiences pénales pour traite et/ou proxénétisme en France, couplées à des entretiens conduits dans le cadre de ProsCrim avec des magistrats (du siège et du parquet) et des avocats (de la défense et de la partie civile), complète cette analyse de la construction pénale de la figure du proxénète en partant du constat d'une prévalence du référentiel culturaliste chez les professionnels du droit ayant à connaître des affaires d'exploitation sexuelle. Face à un phénomène unanimement décrit comme complexe et difficile à « borner » par le droit, magistrats et avocats recourent en effet fréquemment, pour ordonnancer et hiérarchiser les justiciables, à des catégorisations reposant sur l'origine supposée et les schémas délinquants qui lui sont associés. Il s'agit donc ici de comprendre les usages et les effets, sur l'appréhension des faits et la construction du jugement, de ce cadrage culturaliste, dans un système républicain qui se dit par ailleurs aveugle aux différences. Les observations et entretiens réalisés permettent ainsi de poser l'hypothèse, d'une part, que l'argument culturaliste permet à d'autres formes d'attentes prescriptives de se déployer, eu égard notamment au genre et à la sexualité des mis en cause comme des victimes; d'autre part, que l'usage du culturalisme à l'audience vient en appui d'une forme de gouvernement visant, à travers la lutte pénale contre l'exploitation sexuelle, à réguler les frontières tant nationales que morales, sexuelles et raciales.

L'article écrit par Milena Jakšić et Nadège Ragaru, et reposant lui aussi sur des enquêtes conduites dans le cadre du projet ProsCrim, vient élargir encore la réflexion sur la sphère judiciaire comme « arène morale 35 » où l'ordre moral dominant est confirmé et recréé : les demandes de réparation monétaire déposées par les victimes reconnues de la traite des êtres humains devant la Commission d'indemnisation des victimes d'infraction (CIVI) constituent en effet la dernière étape d'un parcours de labellisation institutionnelle des victimes et de reconnaissance de leurs droits, dans le cadre d'une procédure civile succédant à la procédure pénale. À partir d'observations d'audiences, d'entretiens et de consultations de dossiers déposés devant la CIVI, cet article met l'accent sur les attentes pesant sur les victimes et leur conduite de vie, que révèlent les processus d'évaluation auxquelles elles sont ici soumises et qui reposent sur des savoirs tant légaux que médicaux. Tout en contribuant à une analyse du rôle des émotions, des valeurs et des affects dans le travail juridicobureaucratique de production de catégories ${ }^{36}$, l'article de Milena Jakšić et Nadège Ragaru révèle également la centralité de la sexualité, et de sa structure

34. Voir Darley M., «Juger la traite des êtres humains en France et en Allemagne : la construction pénale de la victime d'exploitation sexuelle ", (à paraître), 2022.

35. Komter M. L., "Remorse, Redress, and Reform: Blame-taking in the courtroom", in Travers M., Manzo J.F. (eds.), Law in action: Ethnomethodological and conversation analytic approaches to law, Ashgate, Dartmouth Publishing Co, 1997, pp. 239-264.

36. Graham M., "Emotional Bureaucracies: Emotions, Civil Servants, and Immigrants in the Swedish Welfare State", Ethos, vol. 30, n³, 2002, pp. 199-226 ; Fassin D., "Économies morales et justices locales ", Revue française de sociologie, vol. 53, n 4 , 2012, pp. 651-655. 
genrée sous-jacente, dans l'évaluation de la «bonne vie » des victimes, qui guide la traduction de leur souffrance en argent.

La traduction, proposée en fin de dossier, d'un texte d'Elizabeth Bernstein montre, à partir de plusieurs années d'enquête auprès d'activistes féministes anti-traite aux États-Unis, comment l'approche carcérale est devenue prédominante sur la question des violences sexuelles en général, et de la traite des femmes en particulier. Cette analyse s'inscrit ainsi à la croisée de différents courants de recherche très féconds et cherchant à interroger le rôle du sexe et du genre dans les processus de transformation pénale, d'une part, et les liens entre politiques carcérales et politiques néolibérales, d'autre part. Elizabeth Bernstein propose alors, pour rendre compte de cette double évolution, le concept de "féminisme carcéral », visant à décrire la reformulation en des termes pénaux, voire carcéraux, des luttes engagées pour la libération des femmes, et dont l'activisme anti-traite est particulièrement emblématique. Elle invite à porter à la fois une attention plus soutenue, dans l'analyse des punitivités contemporaines, au recours aux arguments du genre et du sexe en soutien aux politiques carcérales néolibérales, et à intégrer davantage l'État pénal dans les débats et travaux féministes. Ce faisant, le texte d'Elizabeth Bernstein offre une mise en perspective précieuse aux textes réunis dans ce dossier, et qui visent précisément à répondre aux défis analytiques posés par l'autrice. Sont ainsi mis en lumière, au fil des textes, non seulement le virage punitif des politiques des luttes contre la traite, inscrites dans un objectif de préservation de l'ordre public et largement dominées par un objectif de sanction pénale, mais aussi les implicites genrés, sexualisés et racialisés du « féminisme carcéral » qui les sous-tend.

La lutte contre la traite, inscrite dans un objectif de préservation de l'ordre public, apparaît donc au fil des textes proposés comme traversée par de nombreuses projections sur l'appartenance «culturelle » et/ou ethnoraciale, sexuelle et de genre des victimes comme des auteurs. L'étude des différents acteurs (civils ou pénaux, étatiques ou non étatiques) qui font advenir la traite et ses protagonistes, à travers leur participation à l'émergence des catégories (de «traite », de « victime » ou de «prostituée », de «trafiquant» ou de "proxénète " notamment) permettant de penser le phénomène, révèle l'inscription de ces catégories dans une "pensée d'État 37 » articulée autour de la nécessité de tracer des frontières : celles-ci, loin de désigner les seules limites de l'ordre national, révèlent surtout combien la « vérité fondamentale 38 » de l'État-nation qui se donne à voir ici prétend à l'homogénéité non seulement nationale, mais aussi genrée et sexuelle, faisant des politiques de lutte contre la traite des instruments à la croisée entre gouvernement des migrations et gouvernement des sexualités.

37. Sayad A., "Immigration et "pensée d'État" ", Actes de la recherche en sciences sociales, Vol. 129, 1999, pp. 5-14. 
En cela, ce dossier résonne fortement tant avec le texte hors-dossier de Pierre Weiss qu'avec la chronique bibliographique proposée par Fred Salin. Dans le premier en effet, l'invitation à questionner les catégories censées faire communauté, quoique sur un terrain très différent (les clubs de football « communautaires » composés de joueurs turcs en France et en Allemagne), livre une réflexion sur le tracé des frontières entre société majoritaire et minoritaire, entre centre et marges, qui fait écho au processus de préservation d'un ordre national genré et racialisé qui sous-tend les politiques d'immigration en général, et les politiques anti-traite en particulier. La chronique bibliographique proposée à partir des ouvrages de Camille Schmoll 39 et Karen Akoka 40 fournit alors une mise en perspective qui conclut autant qu'elle élargit différents questionnements traversant le dossier : l'accent mis par Camille Schmoll sur l'expérience corporelle de la migration par les femmes, et sur sa nécessaire lecture à la croisée des rapports sociaux de genre et de race, fournit ainsi un éclairage contextuel autant qu'un outil analytique précieux pour penser la complexité des différentes formes d'intrication entre travail du sexe, exploitation sexuelle et migration; la sociologie du travail de jugement de l'asile proposée par Karen Akoka permet quant à elle d'étayer d'une part la compréhension de la formation du jugement, au cœur de la problématique de ce dossier sur les pratiques institutionnelles (notamment judiciaires) de catégorisation et de labellisation, et d'autre part de saisir l'articulation entre les institutions, leur production normative et leurs effets sur les expériences individuelles et subjectives. Surtout, toutes deux offrent une savante « remise en cause de la prétention des politiques migratoires à sauver les femmes ${ }^{41}$ », dont les politiques anti-traite constituent sans doute un exemple des plus paroxystiques.

38. Ibid., p. 6.

39. Schmoll C., Les damnées de la mer. Femmes et frontières en Méditerranée, Paris, La Découverte, 2020.

40. Akoka K., L'asile et l'exil. Une histoire de la distinction réfugiés/migrants, Paris, La Découverte, 2020.

41. Voir F. Salin dans ce numéro. 
